\title{
Các yếu tố digital marketing tác động đến hành vi mua căn hộ chung cư tại thành phố Hồ Chí Minh
}

\section{Digital marketing factors affecting the buying behavior of apartments in Ho Chi Minh City}

\author{
Nguyễn Thị Phụng ${ }^{1 *}$, Nguyễn Thị Hồng Nhung ${ }^{1}$ \\ ${ }^{1}$ Trường Đại học Tài Chính - Kế Toán, Việt Nam \\ "Tác giả liên hệ, Email: nguyenthiphung @tckt.edu.vn
}

THÔNG TIN

DOI: $10.46223 / \mathrm{HCMCOUJS}$. proc.vi.16.1.1855.2021

Ngày nhận: 7/3/2021

Ngày nhận lại: 30/3/2021

Duyệt đăng: 8/4/2021

\section{Tù khóa:}

căn hộ, hành vi mua, digital marketing, social media marketing, content marketing; search engine marketing, online public relations, affiliate marketing, e-mail marketing

\section{Keywords:}

apartments, buying behavior, digital marketing, social media marketing, content marketing; search engine marketing, online public relations, affiliate marketing, e-mail marketing
TÓM TĂT
Nghiên cứu này nhằm phân tích các yếu tố Digital Marketing tác động đến hành vi mua căn hộ chung cư tại thành phố Hồ Chí Minh. Qua thực hiện khảo sát trực tuyến và trực tiếp, nhóm nghiên cứu thu thập thông tin đối với 265 khách hàng về "Tác động Digital Marketing đến hành vi mua căn hộ chung cư tại thành phố Hồ Chí Minh" đến từ các công ty bất động sản. Kết quả phân tích dữ liệu cho thấy có 06 yếu tố tác động đến hành vi mua căn hộ chung cư, đó là Social Media Marketing; Content Marketing; Search Engine Marketing; Online Public Relations; Affiliate Marketing; E-Mail Marketing trong đó yếu tố Social Media Marketing có tác động mạnh nhất đến hành vi mua của khách hàng. Kết quả nghiên cứu sẽ là những thông tin hữu ích cho Doanh nghiệp để khai thác triệt để các phương tiện Digital Marketing nhằm thúc đẩy hành vi mua của người tiêu dùng đối với thị trường căn hộ chung cư tại thành phố Hồ Chí Minh.

\section{ABSTRACT}

This study aims to analyze Digital Marketing factors affecting the buying behavior of apartments in Ho Chi Minh City. Through conducting online and offline, the authors collect information of 265 customers about "the impact of digital marketing on buying behavior of apartments in HCM city" from real estate companies. The results of data analysis show that there are 06 factors affecting the buying behavior of apartments, namely Social Media Marketing; Content Marketing; Search Engine Marketing; Online Public Relations; Affiliate Marketing; E-Mail Marketing. The research results will be useful information for businesses to fully exploit Digital Marketing means to promote consumer buying behavior towards the apartment market in Ho Chi Minh City. 


\section{Giới thiệu}

\section{1. Đặt vấn đề}

Digital Marketing đã tạo nên một cuộc cách mạng về mọi mặt trong cuộc sống của chúng ta. Nó có những tác động đáng kể đối với quy trình kết nối khách hàng của doanh nghiệp nhờ vào sự phát triển vượt bậc của các phương tiện Digital Marketing (Sheoran, 2012).

Digital Marketing là cách thức độc đáo để kết nối với khách hàng thông qua sự tương tác, khả năng đo lường với khách hàng hay các khả năng tùy chỉnh, khả năng truy cập vào các nguồn thông tin mở rộng (Deighton, 1996; Edelman \& Heller, 2015; Merisavo et al., 2007; Salehi, Mirzaei, Aghaei, \& Abyari, 2012). Các marketer đang sử dụng Digital Marketing để chiếm lĩnh thị trường, vì công cụ này có khả năng thu hút, truyền thông cũng như có tầm ảnh hưởng đến người tiêu dùng một cách hấp dẫn nhưng tinh tế nhằm gia tăng sự hài lòng của khách hàng (Gay, Charlesworth \& Esen, 2007). Một sự kết nối để tương tác và nhẳm vào khách hàng mục tiêu thông qua chiến lược Digital Marketing sẽ hiệu quả hơn so với Marketing truyền thống (Hawks, 2015).

Digital Marketing cũng là hình thức để kết nối doanh nghiệp với đúng phân khúc khách hàng mục tiêu. Trong thời đại số hóa, chiến lược tốt nhất của doanh nghiệp là kết nối với khách hàng của mình thông qua môi trường internet - nơi họ dành nhiều thời gian nhất trong ngày.

Hoạt động Digital Marketing có nhiều hình thức khác nhau, tùy theo mục đích của mình thì các doanh nghiệp sẽ lựa chọn hình thức phù hợp. Trong số các hoạt động Digital Marketing hiện nay, doanh nghiệp Việt Nam khá đầu tư và tập trung vào 3 phương tiện đó là: Content Marketing, Social Media Marketing, Search Engine Marketing nhằm thúc đẩy hành vi mua cũng như khơi dậy nhu cầu mua của người tiêu dùng nhờ vào việc sử dụng hiệu quả các phương tiện này.

Theo kết quả khảo sát của $\mathrm{Q} \& \mathrm{Me}$ (2019) về Bức tranh thị trường Digital Marketing Việt Nam 2019 trên 135 doanh nghiệp (102 thương hiệu, 20 agency, 13 công ty truyền thông) cũng chỉ ra rằng: Dẫn đầu là Social Media Marketing với $84 \%$ doanh nghiệp sử dụng hình thức này, theo sau là hình thức Search Engine Marketing và Content Marketing với tỷ lệ sử dụng lần lượt là $52 \%$ và $46 \%$. Có thể nói, Social Media Marketing là hình thức phổ biến với mọi loại hình và quy mô của công ty, đặc biệt là các doanh nghiệp kinh doanh dự án Bất động sản nói chung và căn hộ chung cư nói riêng. Theo thống kê đó, có tới $94 \%$ doanh nghiệp vừa/lớn chi tiêu cho loại hình này, ngoài ra 79\% doanh nghiệp nhỏ cũng thực hiện điều tương tự. Trong đó, những hoạt động Digital Marketing còn lại thường được doanh nghiệp vừa/lớn sử dụng đó là Online Public Relations, Affiliate Marketing, E-Mail Marketing.

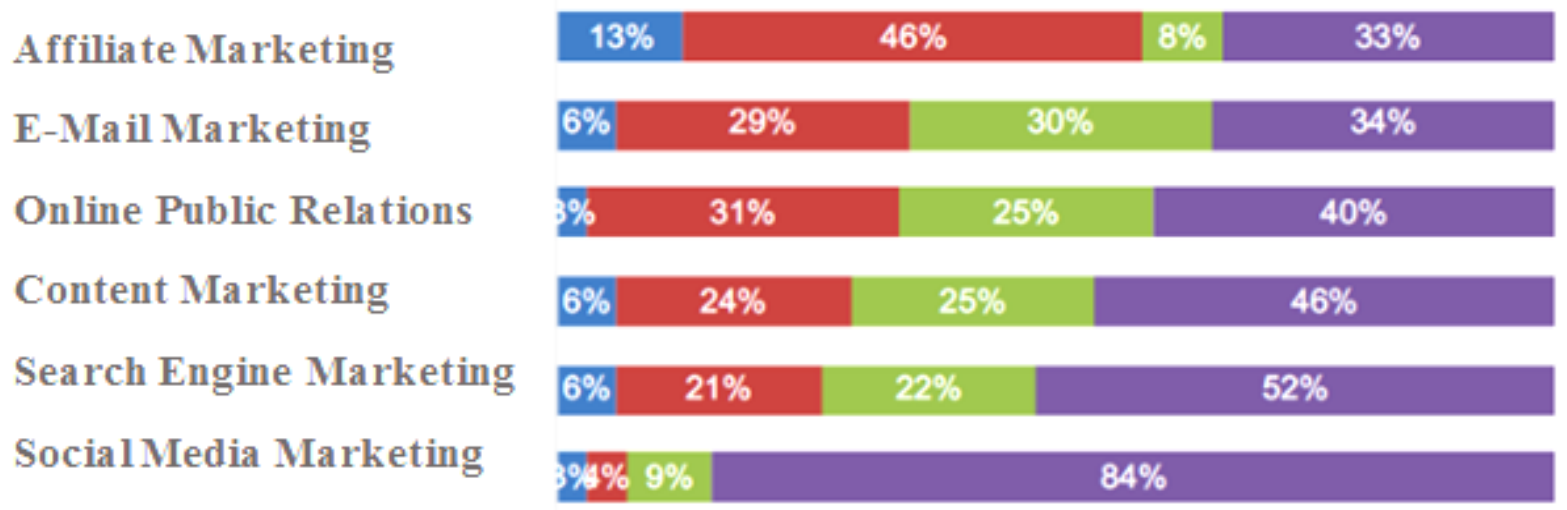

Hình 1. Phản ánh độ phổ biến của các hoạt động Digital Marketing mà doanh nghiệp Việt Nam Nguồn: Q\&Me (2019) 
Kết quả khảo sát dưới đây, cho thấy những ưu điểm lớn nhất mà Digital Marketing mang lại cho doanh nghiệp Việt Nam chính là "tiếp cận khách hàng chính xác hơn $(88 \%)$ ", "đo lường hiệu suất dễ dàng (82\%)" và “quản lý nội dung dễ dàng (81\%).

\section{Advantage of digital marketing}

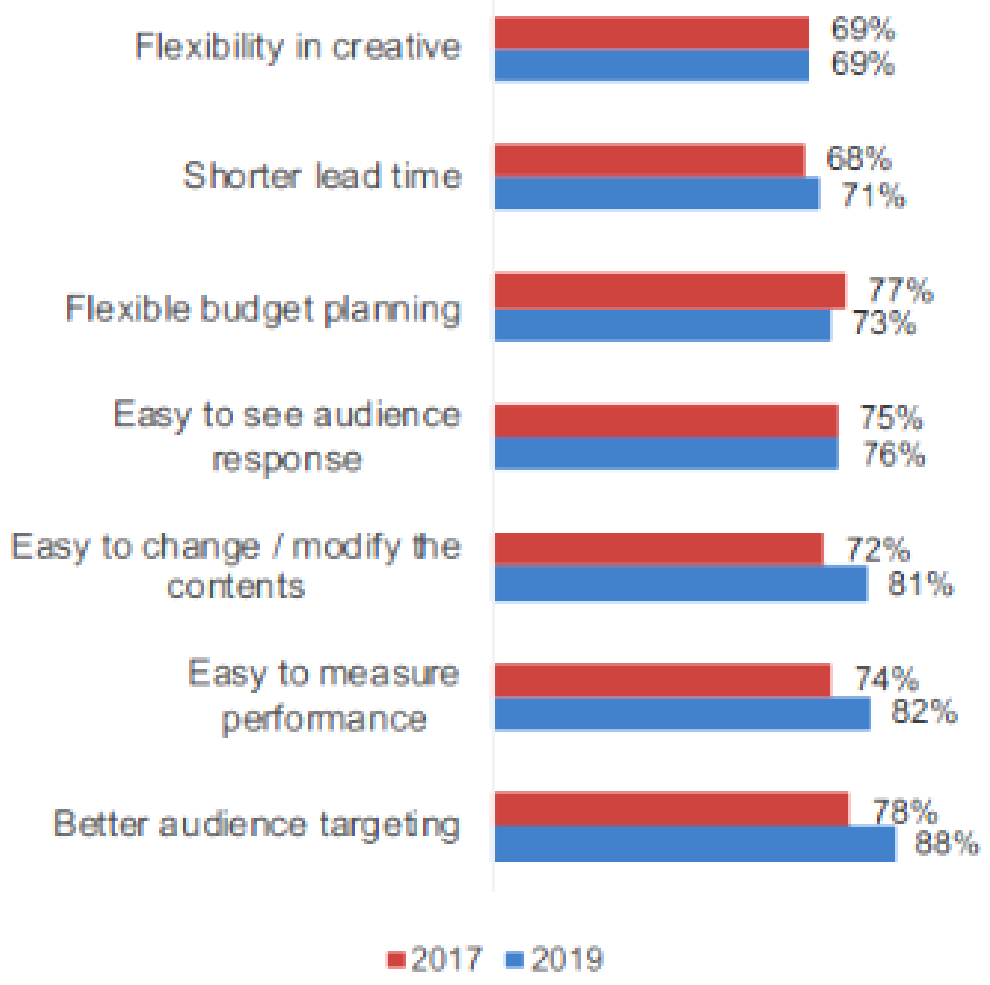

Hình 2. So sánh xu hướng sử dụng các hoạt động Digital Marketing trong 2 năm 2017 và 2019

Nguồn: Q\&Me (2019)

Thị trường Bất động sản nói chung và thị trường căn hộ chung cư nói riêng đang có sự cạnh tranh gay gắt do sự du nhập và phát triển đa dạng của những loại hình khác nhau, hơn nữa hành vi mua của khách hàng cũng trở nên phức tạp hơn. Các nhân viên kinh doanh bắt đầu sử dụng hình thức online để tiếp cận khách hàng chứ không chỉ là tờ rơi, event, hội nghị truyền thống. Chiến lược marketing của Bất động sản tiến lên một tầm cao mới, áp dụng các công cụ, phương tiện của truyền thông, marketing online. Digital Marketing đã tạo nên một xu hướng phát triển mới trong kinh doanh Bất động sản. Digital marketing đem lại hiệu quả vượt trội hơn, phục vụ khách hàng tốt hơn và mang lại lợi nhuận cao hơn.

Với những ưu điểm vượt bậc và đa dạng các phương tiện của Digital Marketing nên doanh nghiệp Bất động sản cần biết cách khai thác triệt để các công cụ này để sử dụng một cách hiệu quả nhằm thúc đẩy hành vi mua hàng của người tiêu dùng trong thời gian sắp tới.

\subsection{Thị trường căn hộ chung cư tại thành phố Hồ Chí Minh}

Theo số liệu thống kê của Q\&Me (2019) về hoạt động Digital Marketing trong ngành Bất động sản bao gồm cả thị trường căn hộ chung cư cho thấy $80 \%$ người mua nhà đều tìm kiếm online, $83 \%$ người mua nhà đều tham khảo ảnh của sản phẩm online, $49 \%$ danh sách khách hàng tiềm năng đến từ việc giới thiệu, $91 \%$ doanh nghiệp Bất động sản dùng social media, $33 \%$ người mua nhà ra quyết định mua lần đầu tiên khi họ tìm kiếm online. 
Thị trường căn hộ tại thành phố Hồ Chí Minh giai đoạn 2010-2019 trở nên khá sôi động và có sự gia tăng mạnh. Số lượng căn hộ được chào bán trong giai đoạn 2015-2019 tăng mạnh (gần gấp ba) so với giai đoạn 2010-2015. Với nguồn cung dồi dào như vậy nhưng số căn hộ được tiêu thụ vẫn tăng đáng kể và vượt hơn nguồn cung được chào bán (giai đoạn 2017-2019). Sự gia tăng như vậy nhờ vào các doanh nghiệp kinh doanh căn hộ thị trường thành phố Hồ Chí Minh tiếp cận khá mạnh mẽ các phương tiện Digital Marketing trong kinh doanh của mình, giúp thúc đẩy hành vi mua của khách hàng cũng như kích thích nhu cầu mua sắm của họ.

\section{Thị trường căn hộ bán TP.HCM, Nguồn cung chào bán và số căn bán được}

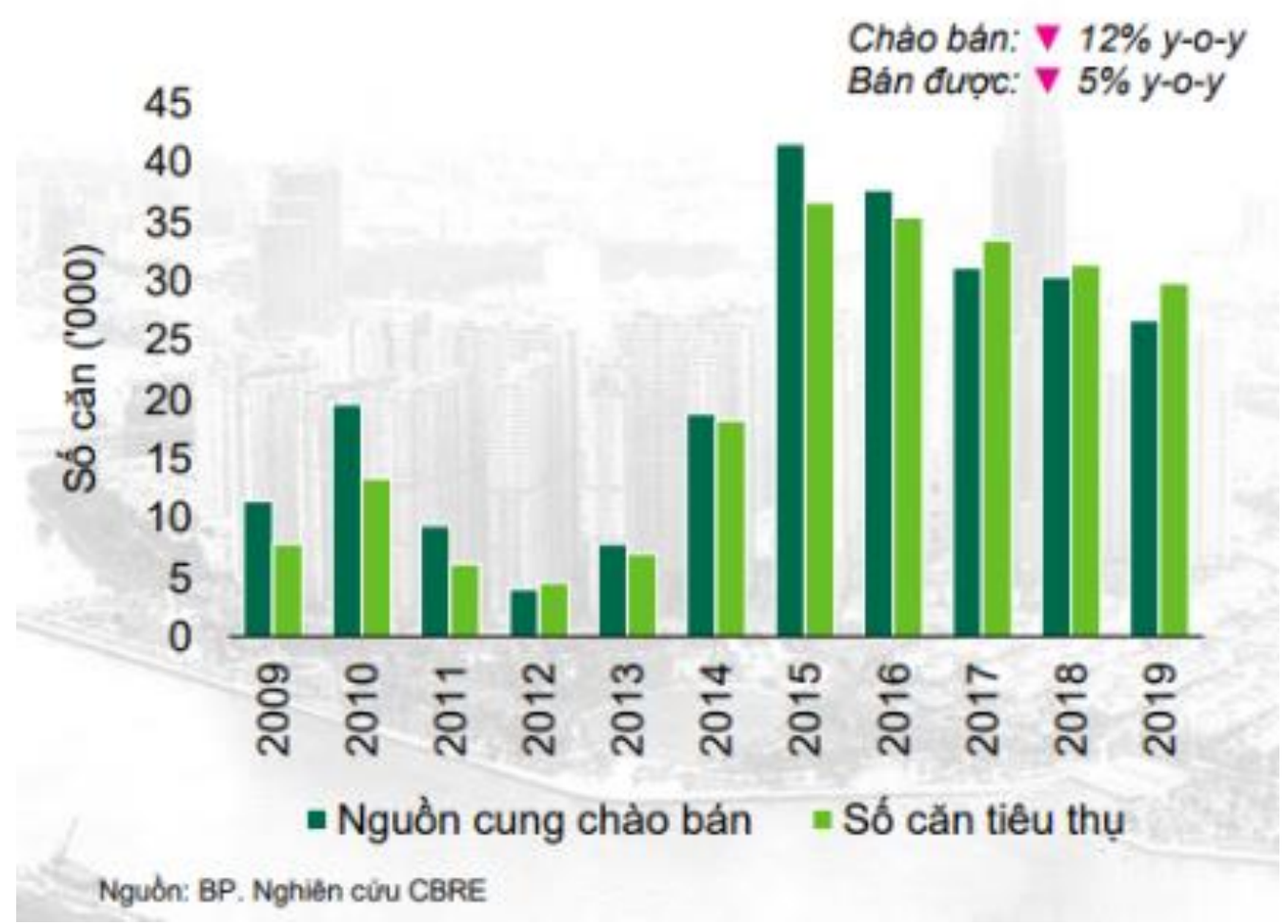

Hình 3. Thị trường căn hộ bán Thành phố Hồ Chí Minh giai đoạn 2009-2019

Nguồn: CBRE (2019)

Trong năm 2020, với tình hình thị trường đang đối mặt với sự ảnh hưởng của dịch bệnh, việc gặp gỡ khách hàng trở nên hạn chế, nhưng các doanh nghiệp không dừng hoạt động của mình mà chuyển sang đẩy mạnh hình thức online với sự phát huy mạnh mẽ của các phương tiện Digital Marketing nên mức độ quan tâm căn hộ chung cư tại TP.HCM vẫn sôi động trên mọi phân khúc. Cụ thể: Theo báo cáo thị trường Bất động sản của Công ty cổ phần DKRA Vietnam, tại thành phố Hồ Chí Minh trong Quý III/2020 có tới 6.374 sản phẩm căn hộ chung cư được mở bán nhưng chỉ tiêu thụ được 5.088 sản phẩm. Ở Quý II có 2.425 sản phẩm chung cư mở bán thì tiêu thụ chỉ được 1.765 sản phẩm và Quý I có 1.547 chung cư mở bán nhưng tiêu thụ được 1.146 căn hộ.

Digital Marketing đã thay đổi cuộc chiến trong lĩnh vực Bất động sản thuộc mảng căn hộ chung cư một cách đáng kể. Ngành Bất động sản ngày trở nên phát triển khi người mua đang hình thành xu hướng tìm kiếm và xem xét căn hộ chung cư trên mạng trước khi ra quyết định mua hàng cuối cùng. Xu hướng này khiến Digital Marketing trở thành một công cụ truyền thông không thể thiếu đối với các doanh nghiệp Bất động sản. 


\section{CĂN Hộ BÁN - TỔNG QUAN TH! TRƯỜNG 2019}

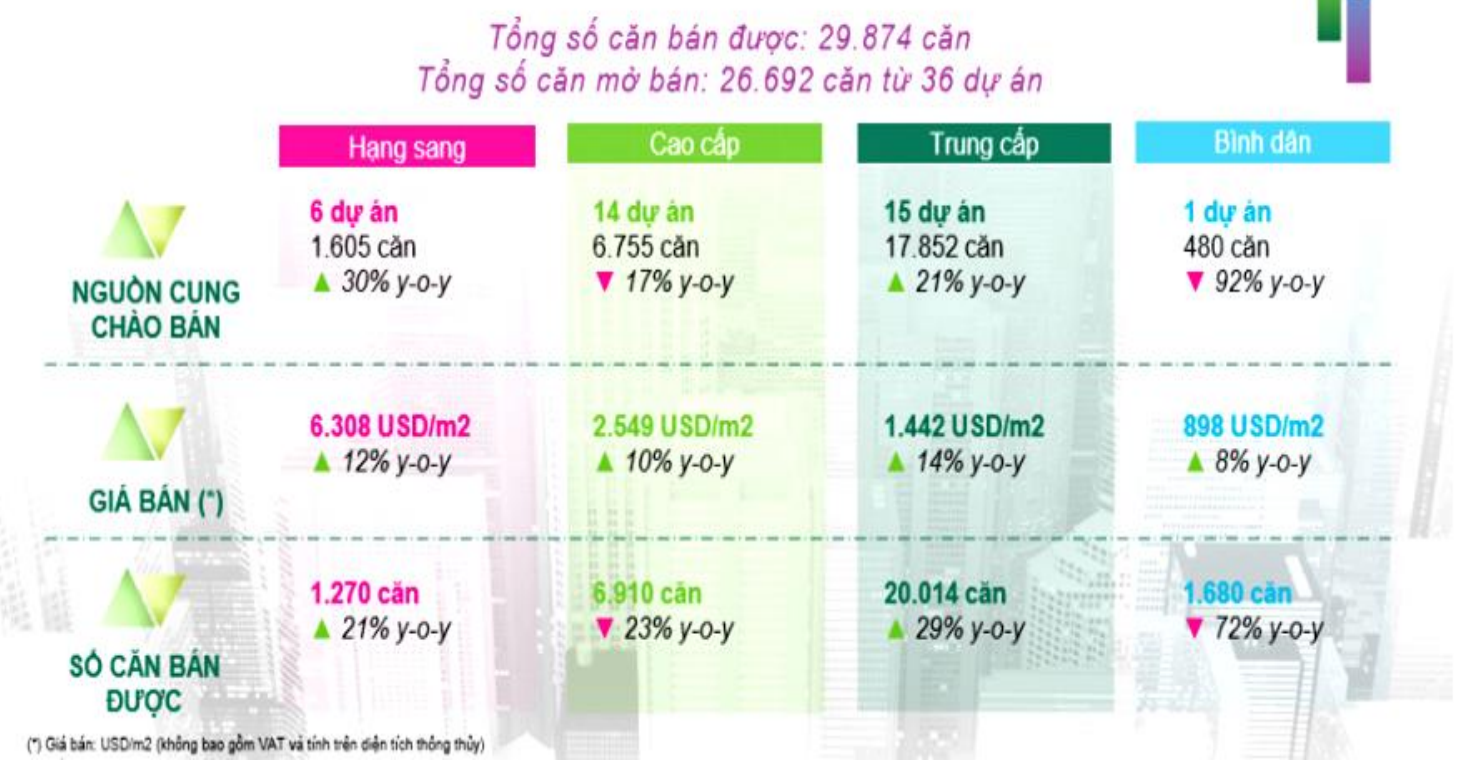

Hình 4. Tổng quan căn hộ bán tại thị trường Thành phố Hồ Chí Minh năm 2019

Nguồn: CBRE (2019)

Một trong những yếu tố quan trọng khiến Digital Marketing trở nên phổ biến trong ngành Bất động sản căn hộ chung cư hiện đại chính nhờ vào sự hiệu quả và chi phí hợp lý. Digital marketing được áp dụng để tối ưu cơ hội tiếp cận người dùng tiềm năng qua các kênh hữu ích. Chính Digital Marketing đã tạo ra một hiện tượng tăng trưởng ngạc nhiên về số lượng lead tiềm năng online thông qua các công cụ marketing. Điều này đã chứng minh tầm quan trọng không thể thiếu của Digital Marketing với các doanh nghiệp Bất động sản hiện nay

\section{Cơ sở lý thuyết và mô hình nghiên cứu}

\subsection{Cơ sở lý thuyết}

\subsubsection{Digital Marketing}

Digital Marketing là phương thức tiếp thị kỹ thuật số nhằm giới thiệu, quảng bá sản phẩm hoặc thương hiệu để tiếp cận đến nhận thức của khách hàng một cách có hiệu quả và tăng hành vi mua hàng của khách hàng Dara (2016). Digital Marketing hoạt động trên một hoặc một vài phương tiện truyền thông internet. Digital Marketing đóng một vai trò rất lớn trong bất kỳ chiến lược marketing nào của doanh nghiệp và đang trở thành yếu tố cốt lõi của chiến lược marketing tổng thể của nhiều tổ chức.

Digital Marketing có vai trò rất quan trọng trong hầu hết các lĩnh vực kinh doanh đặc biệt trong lĩnh vực Bất động sản (Kumar, 2014). Việc ứng dụng Digital Marketing có thể tạo ra nhiều khách hàng tiềm năng và có thể giúp nhân viên kinh doanh liên hệ với đúng khách hàng mục tiêu. Dara (2016) đã kiểm chứng việc sử dụng các phương tiện Digital Marketing để thu hút khách hàng bao gồm: Social Media Marketing, Content Marketing, Search Engine Marketing, Online Public Relations, Affiliate marketing, E-Mail Marketing ảnh hưởng tích cực đến hành vi mua của người tiêu dùng.

\subsubsection{Hành vi тиа}

Hành vi mua là những hoạt động liên quan tới quy trình nhận thức nhu cầu, tìm kiếm, thu 
thập thông tin, thực hiện mua sắm, sở hữu, sử dụng. Nói cách khác hành vi mua của người tiêu dùng là những hành động mà người tiêu dùng thực hiện trong việc tìm kiếm, mua sắm, sử dụng, đánh giá sản phẩm và dịch vụ mà họ mong đợi sẽ đáp ứng mong muốn cá nhân của họ. Theo Kotler và Armstrong (2012) hành vi mua là những hành vi cụ thể của một cá nhân khi thực hiện các quyết định mua sắm, sử dụng và từ bỏ sản phẩm/dịch vụ. Hành vi mua nghiên cứu về quá trình các cá nhân, tổ chức lựa chọn, mua sắm, sử dụng, hay loại bỏ đối với các sản phẩm dịch vụ, ý tưởng cũng như việc trải nghiệm để thỏa mãn các nhu cầu và mong muốn của họ (Solomon, 2017). Tóm lại, hành vi tiêu dùng là những suy nghĩ, cảm nhận và hành động diễn ra trong quá trình ra quyết định mua sắm hàng hóa, dịch vụ của người tiêu dùng dưới những ảnh hưởng qua lại của các tác nhân kích thích từ môi trường bên ngoài và quá trình tâm lý bên trong của họ.

Häubl và Trifts (2000) định nghĩa hành vi mua online là hoạt động mua sắm của người tiêu dùng qua giao diện máy tính và các thiết bị kết nối internet, được kết nối và tương tác với gian hàng điện tử của nhà bán lẻ thông qua internet. Những thuộc tính của môi trường mua hàng online là có sự tương tác cao, có sự trao đổi thông tin, thông tin theo nhu cầu, sự phản hồi ngẫu nhiên, nội dung có thể tùy chỉnh, và sự phản hồi tức thời (Alba et al., 1997; Ariely, 2000; Häubl \& Trifts, 2000; Zack, 1993)

Hành vi mua có thể là lý tính hoặc cảm tính giúp một người được tận hưởng và thỏa mãn nhu cầu, đó cũng là cơ sở giải thích lý do vì sao người ta mua hàng. (Rohm \& Swaminathan, 2004; Sarkar, Butler, \& Steinfield, 1995). Những lợi ích này liên quan đến vị trí, thời gian linh động, thanh toán nhanh và thuận tiện, vận chuyển nhanh và sản phẩm phong phú (Rohm \& Swaminathan, 2004). Chẳng hạn, có thể phân loại người tiêu dùng theo loại động cơ mua sắm thành 6 nhóm, bao gồm nhóm mua sắm thuận tiện giúp tiết kiệm thời gian, nhóm tìm kiếm thông tin, nhóm tương tác xã hội, nhóm mua sắm giải trí, nhóm tìm kiếm nhiều lựa chọn, nhóm khao khát sở hữu ngay sản phẩm.

Lý thuyết này cho thấy việc mua online là mua sắm thông qua internet và thông qua máy tính, các thiết bị kết nối internet. Do đó có những đặc điểm là không tiếp xúc với người bán online mà thông qua môi trường mạng vì vậy mức độ cảm nhận rủi ro cao hơn, và yếu tố lòng tin rất quan trọng ảnh hưởng đến quyết định lựa chọn mua hàng của người tiêu dùng. Từ cơ sở này giúp doanh nghiệp gia tăng chất lượng các phương tiện Digital Marketing là giao diện mà người tiêu dùng tương tác để mua hàng và danh tiếng là yếu tố giúp hình thành sự tin tưởng của khách hàng.

\subsection{Mô hình và giả thuyết nghiên cúu}

Dựa trên các lý thuyết về hành vi mua, các yếu tố ảnh hưởng đến hành vi mua hàng, tác giả đề xuất mô hình nghiên cứu các yếu tố của Digital Marketing tác động đến hành vi mua căn hộ tại thành phố Hồ Chí Minh. Mô hình nghiên cứu này tập trung vào các yếu tố sau:

\section{- Yếu tố "Social Media Marketing"}

Social Media Marketing là quá trình tiếp thị nội dung, thông tin nhằm đạt được lưu lượng truy cập lớn hoặc sự chú ý thông qua các trang mạng xã hội (Krishnamurthy \& Sin, 2014). Các nền tảng Social Media Marketing bao gồm Facebook, twitter, YouTube, LinkedIn, Snapchat, Pinterest, Quora và Instagram, v.v. Các nền tảng này sẽ cung cấp cho các marketers nhiều lựa chọn để tiếp thị sản phẩm của họ bao gồm các dữ liệu doanh nghiệp, các nhóm khách hàng khác nhau hay các thông điệp truyền thông khác nhau. Social Media Marketing ảnh hưởng đến người tiêu dùng dưới nhiều hình thức khác nhau như: Từ bạn bè, các quảng cáo trên mạng xã hội để mua các sản phẩm. Đồng thời, Social Media Marketing giúp mở rộng các mối quan hệ với khách hàng bằng cách tạo sự kết nối với nhau.

Social Media Marketing về cơ bản được nhiều người dùng sử dụng để chia sẻ những quan 
điểm, thành tích của doanh nghiệp, lý do chính dẫn đến sự phổ biến của Social Media Marketing là vì tính kết nối rộng rãi của nó. Tuy nhiên, nếu các doanh nghiệp muốn sử dụng công cụ này để tiếp cận khách hàng một cách hiệu quả thì họ phải trả phí. Khi mọi người kích vào các khuyến mãi này, họ sẽ được chuyển đến trang web của các marketer. Điều này giúp các khách hàng tiềm năng này sẽ có thêm các thông tin về sản phẩm hay dịch vụ mà họ tiềm kiếm, nếu như các sản phẩm và dịch vụ đó đáp ứng được nhu cầu của họ thì họ có thể quyết định mua hàng.

Giả thuyết H1: Yếu tố Social Media Marketing tác động tích cực đến hành vi mua căn hộ chung cư tại thành phố Hồ Chí Minh.

\section{- Yếu tố "Content Marketing"}

Content Marketing là một cách tiếp cận khách hàng có tính chiến lược, điều này có nghĩa là Content Marketing sẽ hàm chứa các đánh giá có giá trị về sản phẩm/ dịch vụ cũng như các thông tin liên quan và nhằm giúp cho khách hàng tiềm năng dễ dàng ra quyết định. Content Marketing được sử dụng khá rộng rãi thông qua các blog sẽ cung cấp nhiều nội dung hữu ích cho khách hàng tiềm năng dựa trên sở thích và dữ liệu nhân khẩu học của họ. (Rakic, Beba, \& Mira, 2014).

Content Marketing rất hiệu quả trong việc sử dụng để thu hút những khách hàng đang tìm kiếm một số sản phẩm để đáp ứng nhu cầu của mình nhưng họ lại đang phân vân trong quá trình chọn lựa. Khi khách hàng xem các blog và bài đăng, nếu như họ thích, họ có thể kích vào các đường link được cung cấp bởi những trang này. Với cách thức đó sẽ gia tăng lưu lượng truy cập đến các website đó nhiều hơn.

Giả thuyết H2: Yếu tố Content Marketing tác động tích cực đến hành vi mua căn hộ chung cư tại thành phố Hồ Chí Minh.

\section{- Yếu tố "Search Engine Marketing"}

Search Engine Marketing được xem như là nhu cầu hàng giờ của đa số các khách hàng. Họ thường tìm kiếm hàng triệu những yêu cầu dưới dạng từ khóa trên các công cụ tìm kiếm để có những thông tin liên quan đến sản phẩm và dịch vụ. Trên thị trường hiện nay có vô số người bán với các mặt hàng đa dạng hoặc cũng có thể bán cùng sản phẩm/ dịch vụ, chính vì vậy các marketer phải đối mặt với sự cạnh tranh rất lớn với các đối thủ.

Search Engine Marketing (SEM) là một loại hình tiếp thị dựa trên Internet liên quan đến việc nghiên cứu, sử dụng nhiều phương pháp Marketing khác nhau nhằm đưa website của doanh nghiệp đứng ở vị trí hàng đầu trong kết quả tìm kiếm trên Internet. Tối ưu hóa công cụ tìm kiếm (SEO) được xem là một phần của SEM. (Nabout, Skiera, Stepanchuk, \& Gerstmeier, 2012). Nó nghiên cứu từ khóa, phân tích cạnh tranh và các dịch vụ công cụ tìm kiếm khác nhằm tăng lưu lượng tìm kiếm đến trang web của doanh nghiệp.

Giả thuyết H3: Yếu tố Search Engine Marketing tác động tích cực đến hành vi mua căn hộ chung cư tại thành phố Hồ Chí Minh.

\section{- Yếu tố "Online Public Relations"}

Online Public Relations nhằm mục tiêu xây dựng và gìn giữ hình ảnh tốt đẹp của doanh nghiệp đối với cộng đồng, hình thức này phụ thuộc vào danh tiếng của nhân vật công chúng và cũng tùy thuộc vào cách thức duy trì danh tiếng của thương hiệu thông qua các phương tiện trực tuyến. Ngày nay, các bài đánh giá và xếp hạng của Google, về cơ bản là hỗ trợ trực tuyến để duy trì mối quan hệ với công chúng. Các nhà bán lẻ duy trì mối quan hệ công chúng bằng cách gửi thư và tin nhắn liên tục cho khách hàng của họ về các sản phẩm mới, ưu đãi và khuyến mãi. Với cách thức này, khách hàng sẽ cảm thấy được kết nối với thương hiệu một cách thân thiết hơn. Online 
Public Relations cũng được quản lý thông qua các nền tảng truyền thông xã hội, messengers và ứng dụng di động (Wolburg, 2010). Quản lý danh tiếng của thương hiệu phụ thuộc vào sự lan truyền trên nền tảng công nghệ số.

Giả thuyết H4: Yếu tố Online Public Relations tác động tích cực đến hành vi mua căn hộ chung cư tại thành phố Hồ Chí Minh.

- Yếu tố "Affiliate Marketing"

Affiliate marketing: Là mô hình quảng bá sản phẩm/ dịch vụ, trong đó các nhà tiếp thị liên kết hoặc các nhà quảng cáo dựa trên năng lực, sự hiểu biết của mình sẽ thực hiện chiến lược Marketing nhằm thu hút và mời chào người ra quyết định mua sản phẩm/ dịch vụ của Nhà cung cấp. Từ đó các nhà tiếp thị liên kết hoặc các nhà quảng cáo sẽ nhận được hoa hồng từ Nhà cung cấp đối với mỗi đơn hàng thành công (Edelman \& Brandi, 2015). Affiliate Marketing tận dụng khả năng của các chủ thể nói trên để có chiến lược marketing hiệu quả hơn.

Giả thuyết H5: Yếu tố Affiliate Marketing tác động tích cực đến hành vi mua căn hộ chung cư tại thành phố Hồ Chí Minh.

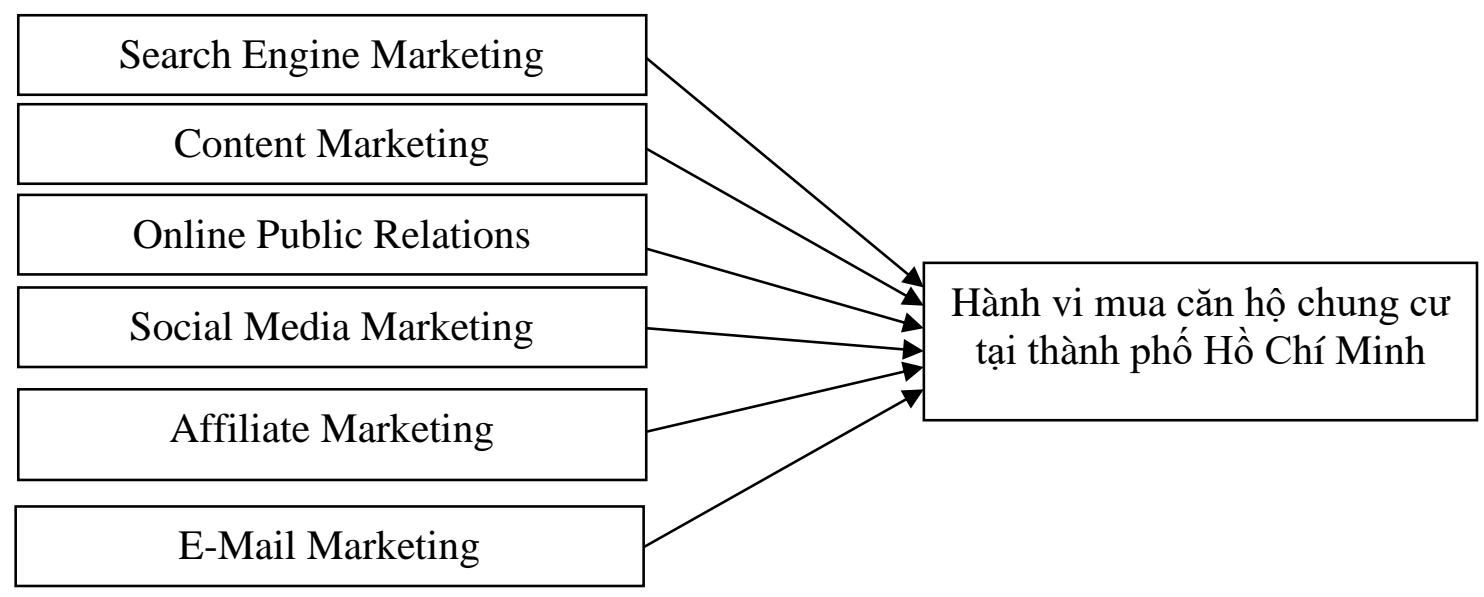

Hình 5. Mô hình nghiên cứu đề xuất

Nguồn: Tác giả đề xuất

\section{- Yếu tố "E-Mail Marketing"}

E-mail marketing là một trong những công cụ Digital Marketing truyền thống. E-mail marketing một lần nữa là database marketing, trong đó cơ sở dữ liệu về e-mail được chuẩn bị bởi các nhà làm online marketing hoặc Digital Marketing và các thư được gửi đến những người có thể quan tâm đến việc mua các sản phẩm (Chadwick \& Doherty, 2012). Có nhiều khía cạnh quan trọng góp phần vào thành công của e-mail marketing như tỷ lệ mở e-mail, trả lời e-mail, khách hàng tiềm năng, truy cập vào đường dẫn của email, v.v. E-mail marketing, để có kết quả nhanh hơn, được thực hiện bởi phần mềm như mail chimp, drip-in, v.v. E-mail marketing có nhiều lợi ích hơn các nền tảng khác như E-Mail có thời gian hiển thị nội dung trong hộp thư đến tốt hơn và khách hàng có thể tham khảo bất kỳ lúc nào trong tương lai. Hơn nữa, nội dung của e-mail phức tạp hơn các nền tảng trực tuyến khác.

Giả thuyết H6: Yếu tố E-Mail Marketing tác động tích cực đến hành vi mua căn hộ chung cư tại thành phố Hồ Chí Minh.

\section{Phương pháp nghiên cứu}

Nghiên cứu này được sử dụng theo phương pháp định lượng. Dữ liệu nghiên cứu được thu 
thập thông qua bảng hỏi sử dụng thang đo 07 điểm (từ hoàn toàn không đồng ý đến hoàn toàn đồng ý). Tổng số bảng hỏi được phát ra là 265 bảng và có thu về 250 bảng hỏi đạt yêu cầu chiếm tỷ lệ 93,34\%

Dữ liệu sau khi làm sạch được tiến hành phân tích đặc điểm mẫu nghiên cứu thông qua các thống kê mô tả, sau đó thực hiện đánh giá độ tin cậy thang đo bằng hệ số Cronbach's Alpha, giá trị thang đo bằng phân tích nhân tố khám phá (EFA), để loại dần các biến có trọng số nhỏ hơn 0,5 . Thang đó được chấp nhận khi giá trị hệ số KMO lớn hơn hoặc bằng 0,5 và nhỏ hơn hoặc bằng 1 (Othman \& Owen, 2002), Eigenvalue lớn hơn 1 và tổng phương sai trích được bằng hoặc lớn hơn 50\% (Gerbing \& Anderson, 1988).

\section{Kết quả nghiên cứu}

\subsection{Phân tích nhân tố và đánh giá độ tin cậy}

Thang đo các yếu tố trong nghiên cứu được tác giả đánh giá bằng hệ số tin cậy Conbach’s Alpha, kết quả kiểm định được trình bày trong bảng 1. Qua phân tích Conbach's Alpha cho thấy các thang đo hệ số Conbach's Alpha lớn hơn 0,6 (0,629 đến 0,866), hệ số tương quan biến tổng cac biến của thang đo đều lớn hơn mức cho phép 0,3 (nhỏ nhất bằng 0,376 ). Do đó các thang đo này đều đạt độ tin cậy (Nunnally \& Burnstein, 1994) và được sử dụng trong bước phân tích nhân tố EFA. Kết quả phân tích nhân tố khám phá EFA của thành phần các yếu tố tác động đến hành vi mua căn hộ chung cư tại thành phố Hồ Chí Minh cho thấy từ 27 biến đo lường thuộc 06 nhân tố trên đã trích vào 06 nhân tố giữ nguyên gốc 06 nhân tố này trong khung nghiên cứu đề xuất tại Eigenvalue $=1,120(>1)$ và phương sai trích $=64,548 \%(>50 \%)$, cho thấy các biến quan sát đều có hệ số tải nhân tố và phần chênh lệch đạt yêu cầu. Bên cạnh đó, hệ số Keiser - Meyer - Olkin (KMO) là 0,872 , có giá trị Sig. rất nhỏ cho thấy kết quả phân tích nhân tố là đáng tin cậy.

\section{Bảng 1}

Kết quả phân tích Cronbach's Alpha và EFA thang đo các yếu tố trong mô hình nghiên cứu

\begin{tabular}{|c|c|c|c|}
\hline Yếu tố & Ký hiệu & $\begin{array}{l}\text { Hệ số Cronbach's } \\
\text { Alpha }\end{array}$ & $\begin{array}{l}\text { Hệ số tải } \\
\text { nhân tố }\end{array}$ \\
\hline Search Engine Marketing & SEM & 0,860 & \\
\hline $\begin{array}{l}\text { Tôi tìm kiếm sản phẩm và thông tin của sản phẩm } \\
\text { thông qua công cụ tìm kiếm, trước khi tiến hành ra } \\
\text { quyết định mua sản phẩm }\end{array}$ & SEM1 & & 0,656 \\
\hline $\begin{array}{l}\text { Tôi thường kích vào đường link có liên quan trên } \\
\text { công cụ tìm kiếm }\end{array}$ & SEM2 & & 0,490 \\
\hline $\begin{array}{l}\text { Thông qua các công cụ tìm kiếm, tôi tìm thấy thông } \\
\text { tin liên quan về các sản phẩm và dịch vụ. }\end{array}$ & SEM3 & & 0,648 \\
\hline $\begin{array}{l}\text { Thông thường, tôi nhấp vào } 3-4 \text { kết quả tìm kiếm } \\
\text { đầu tiên của nội dung tìm kiếm của mình }\end{array}$ & SEM4 & & 0,697 \\
\hline $\begin{array}{l}\text { Công cụ tìm kiếm hiển thị các kết quả có liên quan } \\
\text { nhất ở trên cùng }\end{array}$ & SEM5 & & 0,801 \\
\hline $\begin{array}{l}\text { Thông qua các công cụ tìm kiếm, Tôi không do dự } \\
\text { mua sản phẩm trực tuyến từ nhà bán lẻ }\end{array}$ & SEM6 & & 0,648 \\
\hline
\end{tabular}




\begin{tabular}{|c|c|c|c|}
\hline Yếu tố & Ký hiệu & \begin{tabular}{|c|} 
Hệ số Cronbach's \\
Alpha
\end{tabular} & $\begin{array}{c}\text { Hệ số tải } \\
\text { nhân tố }\end{array}$ \\
\hline Content Marketing & $\mathbf{C M}$ & $\mathbf{0 , 8 3 5}$ & \\
\hline $\begin{array}{l}\text { Tôi thích đọc trực tuyến các nội dung liên quan về } \\
\text { các sản phẩm tôi dự định mua. }\end{array}$ & CM1 & & 0,715 \\
\hline $\begin{array}{l}\text { Tôi thường kích vào đường link "thêm thông tin", } \\
\text { "biết thêm", v.v. Nếu bài đăng / blog thú vị về điều } \\
\text { gì đó. }\end{array}$ & $\mathrm{CM} 2$ & & 0,687 \\
\hline $\begin{array}{l}\text { Tôi thích tìm kiếm thông tin về sản phẩm để so sánh } \\
\text { và lựa chọn sản phẩm }\end{array}$ & CM3 & & 0,766 \\
\hline $\begin{array}{l}\text { Tôi xem các bài đánh giá trên YouTube / Các bài } \\
\text { đánh giá trực tuyến khác về sản phẩm tôi muốn mua }\end{array}$ & CM4 & & 0,695 \\
\hline $\begin{array}{l}\text { Tôi kích vào đường link để được cung cấp thông tin } \\
\text { và đánh giá về sản phẩm trong các blog hoặc các } \\
\text { trang thương mại điện tử. }\end{array}$ & CM5 & & 0,622 \\
\hline Online Public Relations & OPR & $\mathbf{0 , 8 4 8}$ & \\
\hline $\begin{array}{l}\text { Tôi thích xem các tin nhắn, khuyến mãi về sản phẩm } \\
\text { được cá nhân hóa trên thư gửi về E-Mail cá nhân. }\end{array}$ & OPR1 & & 0,777 \\
\hline $\begin{array}{l}\text { Tôi thích những nhà bán lẻ luôn thông báo về sản } \\
\text { phẩm và giúp đưa ra quyết định tốt hơn }\end{array}$ & OPR2 & & 0,771 \\
\hline $\begin{array}{l}\text { Tôi mua sản phẩm từ những cửa hàng trực tuyến vì } \\
\text { họ cung cấp thông tin về sản phẩm một cách hiệu } \\
\text { quả và kịp thời }\end{array}$ & OPR3 & & 0,772 \\
\hline $\begin{array}{l}\text { Tôi thích mua hàng từ những nhà bán lẻ trực tuyến vì } \\
\text { họ cung cầp đầy đủ thông tin về việc mua hàng và trả } \\
\text { hàng }\end{array}$ & OPR4 & & 0,680 \\
\hline Social Media Marketing & SMM & 0,745 & \\
\hline $\begin{array}{l}\text { Tôi là thường xuyên dùng Facebook hoặc ít nhất một } \\
\text { nền tảng truyền thông xã hội khác }\end{array}$ & SMM1 & & 0,599 \\
\hline $\begin{array}{l}\text { Tôi sử dụng mạng xã hội để khám phá các sản phẩm } \\
\text { trên mạng xã hội }\end{array}$ & SMM2 & & 0,799 \\
\hline Tôi thường kích vào quảng cáo trên mạng xã hội & SMM3 & & 0,492 \\
\hline $\begin{array}{l}\text { Tôi truy cập trang web của các nhà bán lẻ trực tuyến } \\
\text { và khám phá thông tin về sản phẩm }\end{array}$ & SMM4 & & 0,554 \\
\hline Affiliate Marketing & AM & 0,866 & \\
\hline $\begin{array}{l}\text { Tôi sử dụng phiếu giảm giá trực tuyến từ các trang } \\
\text { web khác nhau khi mua sản phẩm }\end{array}$ & AM1 & & 0,680 \\
\hline $\begin{array}{l}\text { Tôi nhấp vào link sản phẩm / dịch vụ hoặc các nhà } \\
\text { bán lẻ trực tuyến khi tôi đọc blog để truy cập trang } \\
\text { sản phẩm }\end{array}$ & AM2 & & 0,638 \\
\hline $\begin{array}{l}\text { Tôi nhấp vào đường link sản phẩm / dịch vụ hoặc } \\
\text { các nhà bán lẻ trực tuyến khi tôi xem các đánh giá }\end{array}$ & AM3 & & 0,671 \\
\hline
\end{tabular}




\begin{tabular}{|c|c|c|c|}
\hline Yếu tố & Ký hiệu & $\begin{array}{c}\text { Hệ số Cronbach's } \\
\text { Alpha }\end{array}$ & $\begin{array}{l}\text { Hệ số tải } \\
\text { nhân tố }\end{array}$ \\
\hline \multicolumn{4}{|l|}{ trên YouTube để truy cập vào trang sản phẩm } \\
\hline $\begin{array}{l}\text { Tôi truy cập trang web của sản phẩm bằng cách nhấp } \\
\text { vào đường link được cung cấp bởi các chuyên gia } \\
\text { hoặc cộng đồng }\end{array}$ & AM4 & & 0,725 \\
\hline E-Mail Marketing & EM & $\mathbf{0 , 7 0 0}$ & \\
\hline Tôi đã đọc E-Mail do các nhà bán lẻ trực tuyến gửi & EM1 & & 0,514 \\
\hline $\begin{array}{l}\text { Tôi quan tâm đến các chương trình khuyến mãi qua } \\
\text { e-mail của các nhà bán lẻ trực tuyến liên quan đến } \\
\text { sản phẩm }\end{array}$ & EM2 & & 0,616 \\
\hline $\begin{array}{l}\text { Tôi kích vào đường link của các nhà bán lẻ trực } \\
\text { tuyến thông qua E-Mail do họ gửi }\end{array}$ & EM3 & & 0,799 \\
\hline $\begin{array}{l}\text { E-Mail từ các nhà bán lẻ trực tuyến đôi khi khiến tôi } \\
\text { mua sản phẩm của họ }\end{array}$ & EM4 & & 0,889 \\
\hline $\begin{array}{l}\text { Hành vi mua căn hộ chung cư tại thành phố Hồ } \\
\text { Chí Minh }\end{array}$ & & 0,629 & \\
\hline $\begin{array}{l}\text { Mua sản phẩm bằng công cụ digital MKT là nhanh } \\
\text { nhất }\end{array}$ & CBBRC1 & & 0,587 \\
\hline $\begin{array}{l}\text { Bất kỳ khi nào có thể, tôi sẽ mua hàng thông qua } \\
\text { công cụ digital MKT }\end{array}$ & CBBRC2 & & 0,527 \\
\hline $\begin{array}{l}\text { Tôi giới thiệu cho người khác mua sản phẩm thông } \\
\text { qua công cụ digital MKT }\end{array}$ & CBBRC3 & & 0,623 \\
\hline
\end{tabular}

Nguồn: Kết quả phân tích dữ liệu của tác giả

\subsection{Phân tích hồi quy bội}

\section{Bảng 2}

Kết quả phân tích hồi quy

\begin{tabular}{|l|c|c|c|}
\hline Tên biến & $\begin{array}{c}\text { Hệ số hồi quy chưa } \\
\text { được chuân hóa }\end{array}$ & $\begin{array}{c}\text { Hệ số hồi quy được } \\
\text { chuẩn hóa }\end{array}$ & $\begin{array}{c}\text { Hệ số } \\
\text { Sig. }\end{array}$ \\
\hline Hằng số & 0,253 & & 0,001 \\
\hline Search Engine Marketing & 0,155 & 0,195 & 0,000 \\
\hline Content Marketing & 0,170 & 0,201 & 0,000 \\
\hline Online Public Relations & 0,139 & 0,210 & 0,000 \\
\hline Social Media Marketing & 0,205 & 0,227 & 0,000 \\
\hline Affiliate Marketing & 0,146 & 0,242 & 0,000 \\
\hline E-Mail Marketing & 0,138 & 0,179 & 0,000 \\
\hline$R^{2}=0,776 ; R^{2}$ hiệu chỉnh $=0,776 ;$ Hệ số Durbin - Watson $=1,642$ & \\
\hline
\end{tabular}

Nguồn: Kết quả phân tích dữ liệu của tác giả 
Sau khi thực hiện phân tích nhân tố khám phá, ta tiến hành phân tích hồi quy bội. Kết quả cho thấy giá trị thống kê $\mathrm{F}=144,482$ của mô hình với mức ý nghĩa sig. $=0,000<0,05$, cho thấy mô hình hồi quy tuyến tính bội phù hợp với tập dữ liệu và có thể sử dụng được. Hệ số $\mathrm{R}^{2}$ hiệu chỉnh $=0,776$, chứng tỏ các nhân tố này giải thích được $77,6 \%$ biến thiên của biến phụ thuộc. Hệ số Durbin - Watson của mô hình có giá trị là 1,642 và hệ số phóng đại phương sai VIF $<10$ nên ta kết luận hiện tượng đa cộng tuyến của mô hình là không đáng kể.

Dựa vào kết quả phân tích cho thấy, với một cấp độ thay đổi về nhân tố Search Engine Marketing thì Hành vi mua của khách hàng đối với căn hộ chung cư tại thành phố Hồ Chí Minh sẽ tăng 0,195 cấp độ. Tương tự đối với Content Marketing, Online Public Relations, Social Media Marketing, Affiliate Marketing, E-Mail Marketing.

\section{Kết luận, gợi ý}

Dựa trên kết quả nghiên cứu tác giả đề xuất một số giải pháp nhằm tăng chất lượng các phương tiện Digital Marketing để thúc đầy hành vi mua của người tiêu dùng như sau:

Thứ nhất, xác định khách hàng mục tiêu

Để chiến lược Digital Marketing hiệu quả, việc đầu tiên doanh nghiệp cần làm là phải xác định được tệp khách hàng mục tiêu. Để thu hút sự chú ý của nhóm khách hàng này, doanh nghiệp cần phải cho họ thấy được họ đang nhận được những giá trị cốt lõi nào. Cụ thể:

- Xác định khách hàng mục tiêu thông qua việc nắm rõ các yếu tố nhân khẩu học (demographics) để xác định nhu cầu của khách hàng, và từ dữ liệu được phân tích đó, Chúng ta dễ dàng phát triển chiến lược Digital Marketing dựa trên những hành vi mua của khách hàng.

- Chia nhỏ nhóm đối tượng đó thành nhiều phân khúc khác nhau, khi đó giúp xác định được nội dung truyền tải chặt chẽ hơn, khiến cho mỗi thông điệp chạm đến trái tim từng phân khúc khách hàng tiềm năng riêng biệt một cách hiệu quả nhất.

- Xác định được những nhu cầu riêng mà từng phân khúc khách hàng tiềm năng đang tìm kiếm, và bắt đầu cung cấp cho họ những giá trị phù hợp mà họ đang mong muốn.

Thứ hai, giảm thiểu thời gian mua căn hộ

Các doanh nghiệp cần tạo điều kiện thuận lợi cho khách hàng giảm thiểu thời gian mua căn hộ thông qua xây dựng hệ thống tiếp nhận, xử lý thông tin và trả lời khách hàng một cách nhanh nhất. Các doanh nghiệp cần sử dụng các phần mềm hỗ trợ đảm bảo giao dịch được thực hiện một cách nhanh nhất.

Thứ ba, chi tiết hóa sản phẩm căn hộ chung cư

Việc chi tiết hóa sản phẩm căn hộ chung cư trên website nhằm tạo cho khách hàng nắm rõ thông tin chi tiết về căn hộ chung cư là một trong những yếu tố để thu hút khách hàng. Song song với việc chi tiết hóa sản phẩm căn hộ chung cư cần phải đảm bảo chất lượng nhằm tạo uy tín và niềm tin cho khách hàng. Nội dung các thông tin cần chính xác, đầy đủ để khách hàng hiểu và cảm nhận rõ hơn về sản phẩm căn hộ chung cư tránh tình trạng có sự khác biệt giữa cảm nhận của khách hàng từ thông tin quảng cáo so với thực tế khi nhận hàng.

Thứ tư, kênh truyền thông mạng xã hội

Trước đây, đối với một số doanh nghiệp, social media (kênh truyền thông mạng xã hội) vẫn là một khái niệm tương đối mới. Khi sự phát triển của social media dần bùng nổ, nhiều doanh nghiệp cũng không biết phải làm gì với trang mạng xã hội của mình, họ chỉ biết rằng doanh nghiệp họ nên sử dụng mà thôi. 
Còn trong thời công nghiệp 4.0 hiện nay, nếu thương hiệu của doanh nghiệp chỉ hiện diện trên mạng xã hội không thôi thì nó cũng nhanh chóng bị lu mờ và lãng quên mãi mãi. Điều quan trọng trong thời 4.0 doanh nghiệo cần làm là: (1) nhắm đúng khách hàng mục tiêu, (2) thu hút họ và đồng thời phát triển mạnh các kênh social media, (3) xây dựng website hoặc (4) fanpage chất lượng ... Tất cả để biến các kênh này thành một nguồn khách hàng tiềm năng cho doanh nghiệp.

Các doanh nghiệp phải tạo ra trải nghiệm mua sắm hấp dẫn cho khách hàng với các công nghệ hình ảnh ba chiều (hologram), thực tế ảo (virtual reality) và di động. Sự kết hợp giữa thực và ảo làm gia tăng sự tiện lợi cho khách hàng. Đặc biệt, cách so sánh giá cả sản phẩm của người tiêu dùng cũng diễn ra trên không gian số, đặc biệt là từ điện thoại di động.

Thứ năm, tạo uy tín và bảo mật cho website thương mại điện tử

Website thương mại điện tử phải có chính sách bảo mật thông tin và hướng dẫn khiếu nại hợp lý. Điều nay sẽ giúp người mua tin tưởng khi tìm kiếm thông tin và ít do dự khi đưa ra quyết định mua. Để tránh trường hợp bị hacker, doanh nghiệp cần phải được kiểm soát chặt chẽ và áp dụng các kiểm tra an toàn thông tin ngay từ khi phát triển ứng dụng và sau khi đưa vào cung cấp. Đồng thời, doanh nghiệp thường xuyên tiến hành rà soát và đánh giá lại mức độ an toàn của các hệ thống vì theo thời gian hệ thống thường xuất hiện các lỗ hổng, rủi ro mới. Hiện nay, đa phần các website chưa chú trọng nhiều đến những nguyên tắc này. Một số đơn vị sau khi có được thông tin của khách hàng đã bán lại hoặc để mất vào tay các đối tượng phạm pháp, gây ra nhiều vụ mất cắp tài khoản ngân hàng. Trong nhiều trường hợp, khi cần giải quyết những vấn đề liên quan đến khiếu nại hàng hóa, người mua hàng cũng không có cơ sở pháp lý để bảo vệ quyền lợi của mình, do vậy, họ không tin tưởng vào các website thiếu thông tin về bảo mật.

Thứ sáu, đưa website trên top google

Doanh nghiệp cần tranh thứ tự website trên top google giống như doanh nghiệp sở hữu vị trí mặt tiền đắt giá. Trong thời đại bùng nổ internet, mỗi doanh nghiệp cần tạo website cho riêng mình. Điều này vô cùng cần thiết và quan trọng. Có thể nói rằng, website chính là bộ mặt của doanh nghiệp.

Website cần được xây dựng hệ thống content và backlink chất lượng. Từ đó, website được cải thiện vị trí trên công cụ tìm kiếm. Khi đó, sản phẩm của doanh nghiệp sẽ tiếp cận khách hàng nhanh chóng và thành công.

Thứ bảy, tạo ra Content tốt trong Marketing căn hộ chung cư

Để viết được một content tốt doanh nghiệp cần chú trọng vào các yếu tố sau: Content phải gây được sự chú ý, thương hiệu phải được nhắc đến trong content, thúc đẩy động cơ mua hàng cho khách hàng.

\section{Thứ tám, cập nhật xu hướng Digital Marketing mới nhất}

Trong những năm qua, lĩnh vực Digital Marketing đã có những bước thay đổi mạnh mẽ. Doanh nghiệp cần đầu tư nhiều thời gian để tìm hiểu về những xu thế phát triển mới nhất trong ngành từ các blog marketing trong nước hoặc nước ngoài để học tập những cách làm Digital Marketing thành công của những thương hiệu lớn trên thế giới. Đồng thời, doanh nghiệp hãy cập nhật thường xuyên những tin tức mới trong ngày, từ tin thời sự cho đến những câu chuyện nhỏ nhưng được lan truyền phổ biến trên mạng. Tất cả những thông tin doanh nghiệp thu được đều có thể chuyển thành ý tưởng sáng tạo hoặc cũng có thể là chất liệu chính để làm một chiến dịch Digital Marketing đạt hiệu quả nhất. 


\section{Tài liệu tham khảo}

Alba, J., Lynch, J., Weitz, B., Janiszewski, C., Lutz, R., Sawyer, A., \& Wood, S. (1997). Interactive home shopping: Consumer, retailer, and manufacturer incentives to participate in electronic marketplaces. Journal of Marketing, 61(3), 38-53. doi:10.2307/1251788

Ariely, D. (2000). Controlling the information flow: Effects on consumers' decision making and preferences. Journal of Consumer Research, 27(2), 233-248. doi:10.1086/314322

Bakshi, G., \& Gupta, S. K. (2015). Impact of online and conventional advertisement on consumer buying behaviour of branded garments. Asian Journal of Management Sciences \& Education, 125-135.

Chadwick, F. E., \& Doherty, N. F. (2012). Web advertising: The role of e-mail marketing. Journal of Consumer Marketing, 65(6), 843-848. doi:10.1016/j.jbusres.2011.01.005

Dara, S. (2016). Effectiveness of digital marketing strategies. International Journal For Innovative Research In Multidisciplinary Field., 2(2).

Edelman, B., \& Brandi, W. (2015). Risk, information, and incentives in online affiliate marketing. Journal of Consumer Marketing, 52(1), 1-12. doi:10.1509/jmr.13.0472

Edelman, D., \& Heller, J. (2015, August 12). How digital marketing operations can transform business. Retrieved October 10, 2018, from McKinsey \& Company website: https://www.mckinsey.com/business-functions/marketing-and-sales/our-insights/howdigital-marketing-operations-can-transform-business

Gay, R., Charlesworth, A., \& Esen, R. (2007). On-line marketing: A customer led approach. Oxford, UK: Oxford University Press.

Häubl, G., \& Trifts, V. (2000). Consumer decision making in online shopping environments: The effects of interactive decision aids. Marketing Science, 19(1), 4-21. doi: $10.1287 / \mathrm{mksc}$.19.1.4.15178

Hawks, M. (2015). Why digital marketing is really people marketing? Retrieved October 11, 2018, from http://www.huffingtonpost.com/mark-hawks/why-digital-marketingis_b_8186574.ht $\mathrm{ml}$ ?ir=India\&adsSiteOverride=

Kotler, P., \& Armstrong, G. (2012). Principles of marketing (14th a.b). London, UK: Pearson Education Limited.

Krishnamurthy, K., \& Sin, E. A. (2014). Social media and its impact on Consumers behavior. International Journal of Economic Practices and Theories, 295-303.

Merisavo, M., Kajalo, S., Karjaluoto, H., Virtanen, V., Salmenkivi, S., Raulas, M., \& Leppäniemi, M. (2007). An empirical study of the drivers of consumer acceptance of mobile advertising. Journal of Interactive Advertising, 7(2), 41-50.

Nabout, N. A., Skiera, B., Stepanchuk, T., \& Gerstmeier, E. (2012). An analysis of the profitability of fee-based compensation plans for search engine marketing. International Journal of Research in Marketing, 29, 68-80.

Nunnally, J. C., \& Burnstein. (1994). Psychometric theory (3rd ed.). New York, NY: McGraw Hill.

O'Neil, M. A., \& Palmer, A. (2004). Importance-performance analysis: A useful tool for directing continuous quality improvement in higher education. Quality Assurance in Education, 12(1), $39-52$.

Othman, A., \& Owen, L. (2002). The multi dimensionality of carter model to measure customer service quality in Islamic banking industry: A study in Kuwait finance house. International 
Journal of Islamic Financial Services, 3(4), 1-12.

Rakic, R., Beba, R., \& Mira, R. (2014). Digital content marketing for organizations as buyers, Ekonomika. Journal of Economic Theory and Practice and Social Issues, 1, 109-123.

Rohm, A. J., \& Swaminathan, V. (2004). A typology of online shoppers based on shopping motivations. Journal of Business Research, 57(7), 748-757. doi:10.1016/S01482963(02)00351-X

Ryan, D. (2014). Understanding Digital Marketing: Marketing strategies for engaging the digital generation (3rd ed.). London, UK: Kogan Page.

Salehi, M., Mirzaei, H., Aghaei, M., \& Abyari, M. (2012). Dissimilarity of e-marketing vs traditional marketing. International Journal of Academic Research in Business and Social Sciences, 2(1), 510.

Sarkar, M. B., Butler, B., \& Steinfield, C. (1995). Intermediaries and cybermediaries: A continuing role for mediating players in the electronic marketplace. Journal of Computer-Mediated Communication, 1(3), 1-14. doi:10.1111/j.1083-6101.1995.tb00167.x

Sheoran, J. (2012). Technological advancement and changing paradigm of organizational communication. International Journal of Scientific and Research Publications, 2(12), 1-6.

Solomon, M. R. (2017). Consumer behaviour: Buying, having, being. London, UK: Pearson Education Limited.

Tiago, M. T. P. M. B., \& Veríssimo, J. M. C. (2014). Digital marketing and social media: Why bother? Business Horizons, 57(6), 703-708. doi:10.1016/j.bushor.2014.07.002

Wolburg, J. M. (2010). Online public relations: A practical guide to developing an online strategy in the world of social media. Journal of Consumer Marketing, 27(5), 481482. doi:10.1108/07363761011063402

Zack, M. H. (1993). Interactivity and communication mode choice in ongoing management groups. Information Systems Research, 4(3), 207-239. doi:10.1287/isre.4.3.207 